Available online @ https://jjem.jnnce.ac.in https:www.doi.org/10.37314/JJEM.2020.040101 Indexed in International Scientific Indiexing (ISI) Impact factor: 1.025 for 2018-19 Published on: 30 November 2020

\title{
Numerical Investigation of Natural Convection and Radiation In a Moving Radial Porous Fin
}

\author{
B.J.Gireesha, G.Sowmya, M.Nikitha \\ Department of P.G. Studies and Research in Mathematics, Kuvempu University, Shankaraghatta, Shimoga, \\ Karnataka, India. \\ Department of Mathematics, Christ University (Deemed to be University), Bangalore, Karnataka, India. \\ bjgireesu@rediffmail.com,g.sowmya34@gmail.com,nikitha.m@maths.christuniversity.in
}

\begin{abstract}
The analysis of the heat transfer of the moving porous radial fin with natural convection and radiation effect has been carried out. Here, Newton's law of cooling, Fourier's law of conduction and Stefan-Boltzmann's law of radiation were considered to formulate the fin model. The generated governing equation was solved numerically using shooting technique based the fourth-fifth order Runge Kutta Fehlberg method. The resulting dimensionless parameters affecting the thermal performance of the fin were analyzed through the graphs. It is observed that, with the improvement of the convective parameter, there is a greater heat transfer from the surface of the fin.
\end{abstract}

Keywords: Natural convection; radiation; thermal analysis; radial fin; moving porous fin.

\section{INTRODUCTION}

The fin is the extended surface, which basically aims to increase the rate of heat transfer between the surface and the environment. The annular fin is used in the liquid gas heat exchanger system. In many engineering applications like aircraft engines, car radiator, gas turbines, automobiles, electrical chips, Hydrogen fuel cell, heat exchangers in power plants etc., fins are widely used to improve heat transfer. Some of the significant works on fin are as follows; Aziz and Khani [1,2] have developed the highly accurate analyticalsolution for the analysis of thermal performance of radial fin using Homotopy Analysis Method (HAM). Peng and Chen [11] showed dominant role played by convective heat transfer for heat dissipation under convection-radiation condition using hybrid numerical technique. The analytical solution of radiation heat loss from top and bottom surface of porous radial fin is given by Darvishi et al. [3, 4]. Further, to predict temperature distribution in aluminium porous fin, Hatami and Ganji [6] used Least Square Method (LSM) and fourth order Runge-Kutta method. They also examined fin efficiency. Hatami et al. [7] have confirmed from the results that, in order to get high refrigeration efficiency, Lewis number should be more than unity. Also to get high fin efficiency, Rayleigh number and porosity has to be increased and mass transfer has to be limited. Darvishi et al. [8] have obtained numerical results from comparison made with closed form of analytical solution for constant thermal conductivity. Heat transfer with natural convection and radiation effect on fully wet porous radial fin has been considered by Khani et al. [8]. Here Spectral Collocation Method is used to solve non-dimensionalized ordinary differential equation involving three highly non-linear terms.

When fin is moved, it generates thrust. When the fin is lifted it is being pushed in opposite direction from water or air which is in motion. To generate torque and power in 
turbines as well as in propellers for translating torqueing force to lateral thrust moving fins are used. Kim et al. [9] have demonstrated a new type of fan-integrated heat sink named as scroll heat sink. Heat dissipation and fluid pumping occurs simultaneously without any space for fan module which is its remarkable feature. To generate an analytical solution for heat transfer, Aziz and Khani [2] applied Homotopy Analysis Method for moving fin of variable thermal conductivity. Here moving fin is losing heat by simultaneous convection and radiation to its surrounding. Torabi et al. [13] have used Differential Transform Method (DTM) to solve the problem of fin moving with constant velocity. Heat transfer in fin with longitudinal rectangular profile was carried out using DTM by Dogonchi and Ganji [5]. From the results it is shown that, higher values of Peclet number and radiation-conduction parameter is desired, if cooling is required. Later on, Ma et al. [10] studied heat transfer in moving porous fins of trapezoidal, convex parabolic and concave parabolic profiles to solve coupled conductive, convective and radiative heat transfer using Spectral Element Method. Different other methods and techniques has been used by researchers. Modified decomposition method (MADM) is used by Roy et al. [12] for longitudinal moving fin to find solutions of singular nonlinear heat transfer equations. Turkyilmazoglu [14] has considered the heat transfer in exponential fin under the influence of convection and radiation. He has also analyzed and compared the effects of Peclet number due to fin movement and heat generation parameter due to heat source.

Based on the above literature survey, we made an attempt to analyze the influence of natural convection and radiation condition on the temperature performance of the porous fin of radial profile. Here, the fin moves with the constant velocity. The formulation has been non-dimenionalised and numerically solve with the utilization of Runge Kutta Fehlberg method. The impact of parameters on the thermal behavior of the fin has been discussed graphically.

\section{Mathematical formulation}

Consider the thermal processing of a radial porous fin of base radius, tip radius, thickness and moving with constant velocity as shown in figure 1.

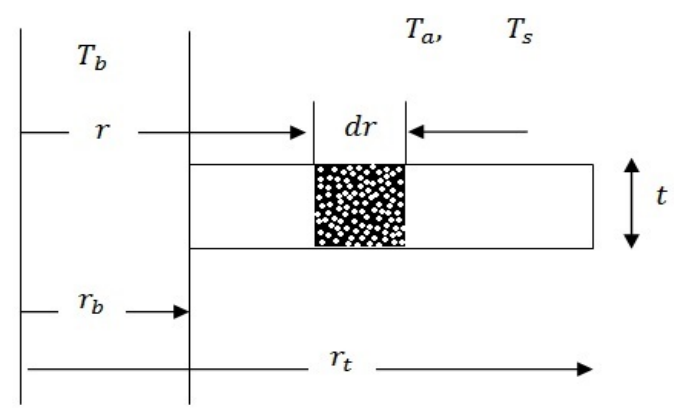

Figure 1: The geometry of the present problem.

Let be the fixed temperature maintained at the base of a fin. Considering as the constant emissivity, when the surface is exposed. Whenever the surface is exposed to colder surrounding medium, it loses heat by convection and radiation. When only natural convection or absent then the radiative component play a prominent role. Thermal conductivity of fin is represented as, fluid has specific heat and density. For convection and radiation, sink temperatures are assumed as and respectively. For the simplification of the model it is assumed that the porous fin is homogeneous, isotropic and saturated with the single-phase fluid, the temperature variation across fin thickness is neglected, the porous medium and the clear fluid interaction are described by the Darcy's formulation, the fin operates under steady state condition. It is also assumed that the heat transfer co-efficient over entire surface of moving material is as a function of temperature and it is as defined as below;

$$
h=h_{a}\left(\frac{T-T_{a}}{T_{b}-T_{a}}\right)^{n},
$$

where, $h_{a}$ is the convective heat transfer coefficient at temperature and is the exponent associated with it.

$q_{\text {rad }}=\sigma \epsilon\left(T^{4}-T_{s}^{4}\right) 2 \pi r d r$

From Newton's law of cooling, rate of cooling 
is given by,

$$
q_{\text {conv }}=h\left(T-T_{a}\right) 2 \pi r d r
$$

Making an energy balance on the element $d r$ of the fin we get,

$\frac{d}{d r}\left(2 \pi r k_{e f f} \frac{d T}{d r}\right) d r=h(1-\phi)\left(T-T_{a}\right) 2 \pi r d r+\sigma \varepsilon\left(T^{4}-T_{s}^{4}\right) 2 \pi r d r$

$+\rho C_{p} 2 \pi r t d r U \frac{d T}{d r}$.

On applying equation (1) in (4) and on simplification we get,

$\frac{1}{r} \frac{d}{d r}\left(r \frac{d T}{d r}\right)-\frac{h_{b}(1-\phi)}{k_{e f f} t} \frac{\left(T-T_{a}\right)^{n+1}}{\left(T_{b}-T_{a}\right)^{n}}-\frac{\sigma \varepsilon\left(T^{4}-T_{s}^{4}\right)}{k_{e f f} t}-\frac{\rho C_{p} U}{k_{e f f}} \frac{d T}{d r}=0 .(5)$

The relevant boundary condition for the finite length fin with insulated tip is,

$$
\begin{aligned}
& \text { at } r=r_{b}, T=T_{b}, \\
& \text { at } r=r_{t}, \frac{d T}{d r}=0 .
\end{aligned}
$$

The dimensionless terms like

$\theta=\frac{T}{T_{b}}, \theta_{a}=\frac{T_{a}}{T_{b}}, \theta_{s}=\frac{T_{s}}{T_{b}}, R=\frac{r}{r_{b}}, N c=\frac{h_{b}(1-\phi) T_{b} r_{b}^{2}}{k_{e f f} t}$,

$P e=\frac{\rho r_{b} C_{p} U}{k_{e f f}}, N r=\frac{\varepsilon \sigma r_{b}^{2} T_{b}^{3}}{k_{e f f} t}$,

are implemented to non-dimensionalize the differential equation (5). Then it recast in the dimensionless form as,

$\frac{1}{R} \frac{d}{d R}\left(R \frac{d \theta}{d R}\right)-N c \frac{\left(\theta-\theta_{a}\right)^{n+1}}{\left(1-\theta_{a}\right)^{n}}-N r\left(\theta^{4}-\theta_{s}^{4}\right)-P e \frac{d \theta}{d R}=0$.

The boundary condition is also non-dimensionalized as

at $R=1, \theta(R)=1$,

at $R=R^{*}, \frac{d \theta(R)}{d R}=0$,

where, $R^{*}$ is the ratio of tip radius to base radius. The dimensionless parameters which impact on the thermal behavior of the radial porous fin are as follows: convective parameter $(N c)$, radiative parameter $(N r)$, Peclet number $(P e)$, exponent $(n)$, dimensionless convective sink temperature $\left(\theta_{a}\right)$ and radiative sink temperature $\left(\theta_{s}\right)$.

\section{Numerical Procedure}

Equation (8) is the non-linear second order ordinary differential equation with the boundary condition (9). Initially, this boundary value problem has been converted to initial value problem. For that we assume,

$$
\theta=x_{1}, \theta^{\prime}=x_{2} \text {. }
$$

Then equation (8) becomes,

$x_{2}{ }^{\prime}=N c \frac{\left(x_{1}-\theta_{a}\right)^{n+1}}{\left(1-\theta_{a}\right)^{n}}+N r\left(x_{1}^{4}-\theta_{s}^{4}\right)+x_{2} P e-\frac{x_{2}}{R}$.

Corresponding initial conditions are:

$$
x_{1}(0)=1, x_{2}(0)=m_{1},
$$

where $m_{1}$ is the unknown and it is determined by Shooting scheme. Then the reduced initial value problem has been solved by the application of Runge-Kutta-Fehlberg fourth fifth order method. Here, we consider the step size as 0.001 and sixth order convergence criteria have been implemented.

\section{Results and Discussion}

The thermal analysis of a moving radial porous fin with natural convection and radiation effect is studied numerically. The effects of different parameters have been plotted by keeping $N c=10, N r=5, R^{*}=2$, $P e=1, \theta_{a}=0.2, \theta_{s}=0.2$ and $n=1$. The plotted graphs have been interpreted as mentioned below. Figure 2 shows the influence of convective parameter on the temperature profile.

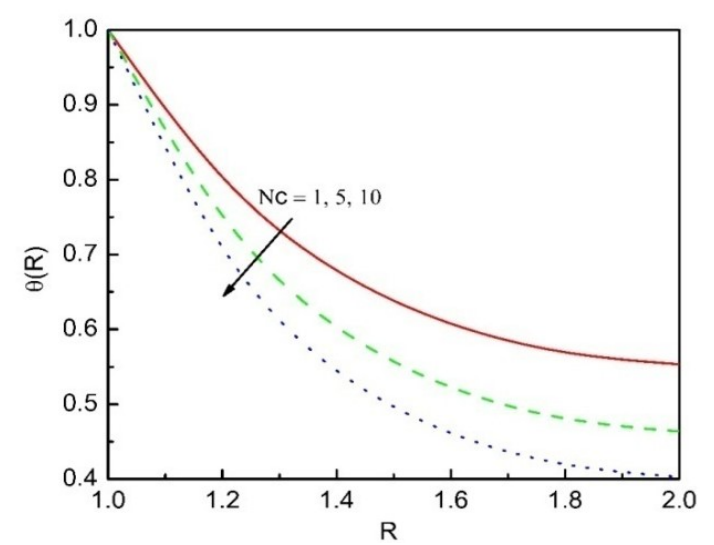

Figure 2 : The influence of convective parameter $(N c)$ on the temperature profile

From this plot one can see that, as $N c$ increases, temperature profile decreases. This 
is obvious from the fact that, as the convection effect increases, it carries more heat from the fin surface. Hence, the temperature of the fin decreases.

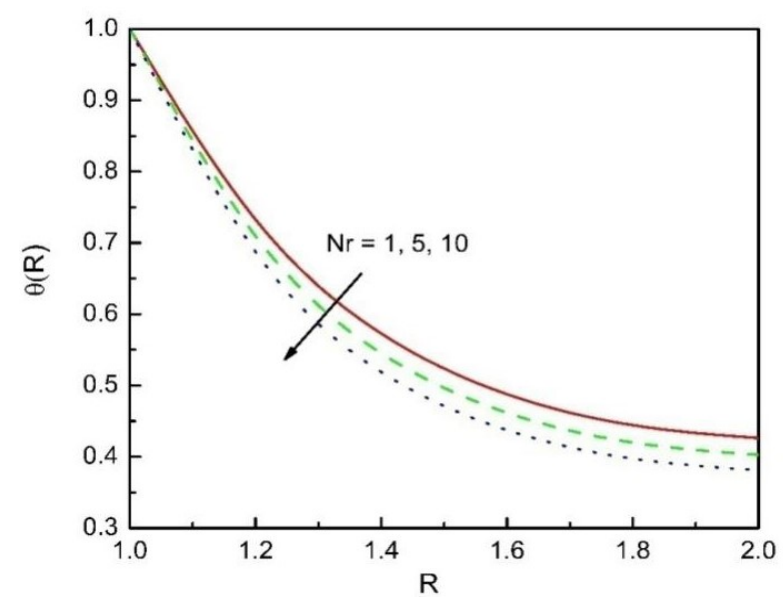

Figure 3 : The influence of radiative parameter $(\mathrm{Nr})$ on the temperature profile

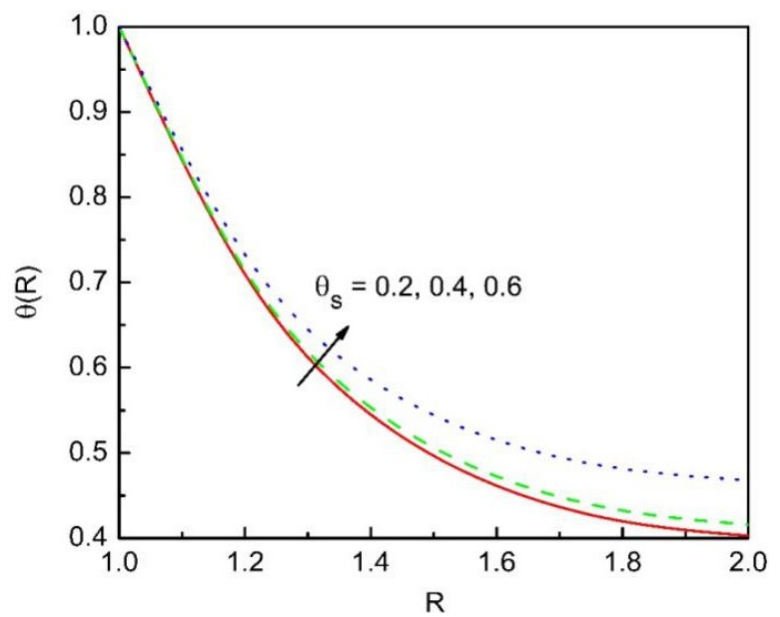

Figure 4 : The influence of dimensionless radiative sink temperature $\left(\theta_{S}\right)$ on the temperature profile

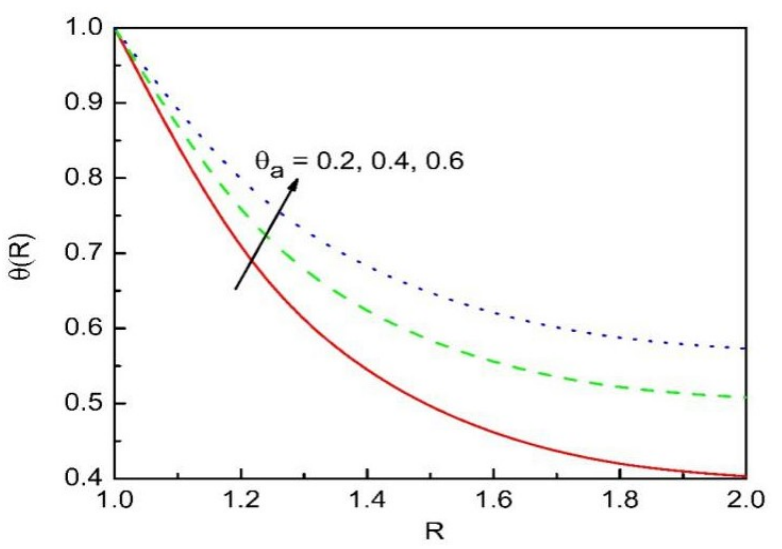

Figure 5 : The influence of dimensionless convective sink temperature $\left(\theta_{a}\right)$ on the temperature profile

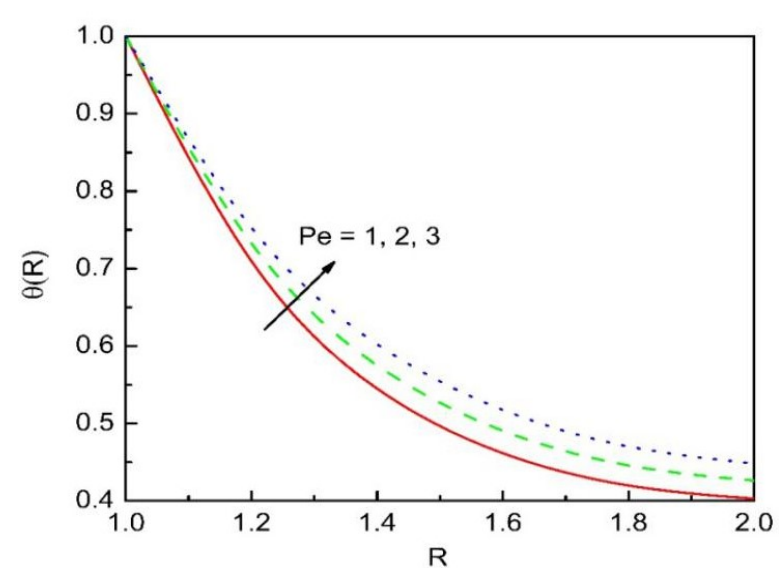

Figure 6 : The influence of Peclet number $(P e)$ on the temperature profile

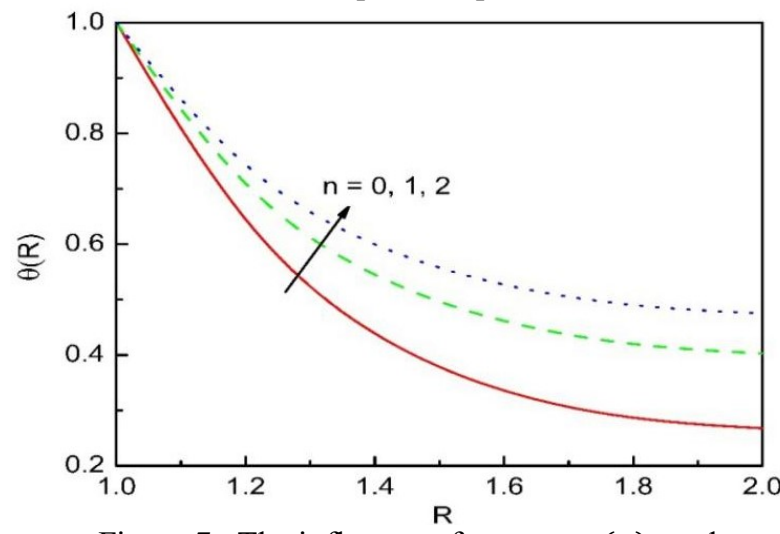

Figure 7 : The influence of exponent $(n)$ on the temperature profile

In figure 3 , effects of radiation parameter $(\mathrm{Nr})$ on thermal distribution is plotted. In this graph, as $\mathrm{Nr}$ increases, temperature profile decreases, due to radiation. Therefore, radiation parameter plays an important role in cooling the system and it proves that radiation parameter effectively controls the temperature. The influence of dimensionless radiative sink temperature on the thermal performance is as represented in figure 4. As $\theta_{s}$ is increased, temperature profile also increases. In figure 5 , impact of varying dimensionless convective sink temperature $\left(\theta_{a}\right)$ on thermal performance of the fin has been shown. It can be seen that temperature profile increases as $\theta_{a}$ increases. Since, the rise in sink temperature decelerates the heat flow from fin surface to the surrounding.

In figure 6, temperature profile for different values of Peclet number has been 
plotted. Here, as the $P e$ rises, temperature profile also increases. This is due to the fact that, as the speed of the fin increases, interactive time between the surface and surrounding declines, hence we observe more temperature on the fin surface. The impact of exponent $n$ on the thermal distribution is as shown in figure 7. It can be noticed that as the value of $n$ increases, temperature profile also increases.

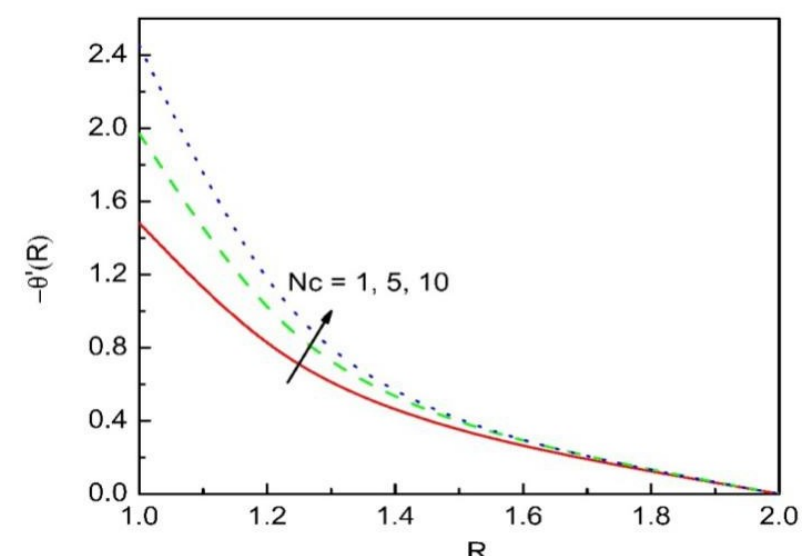

Figure 8 : The influence of $N C$ on the temperature gradient

The effect of $N c$ and $N r$ on the temperature gradient of the fin are as depicted in figures 8 and 9 respectively. It is observed that, as the $N c$ and $N r$ rises, temperature gradient also enhances due to augment in natural convection and radiation environment. Hence, higher values of these parameters improve the heat transfer rate. Figures 10 and 11 shows the influence of radiative and convective sink temperatures on the thermal gradient, respectively. With the rise in sink temperature, gradient shows decreasing result because of reduction in temperature difference between the fin and sounding. So, the lesser value of sink parameter helps in more rate of heat transfer from the fin. The impact of $P e$ and $n$ on the temperature gradient is presented in figures 12 and 13 respectively.

As $P e$ and $n$ enhances, temperature gradient decreases due to an increase in velocity of fin as well as nonlinearity respectively.

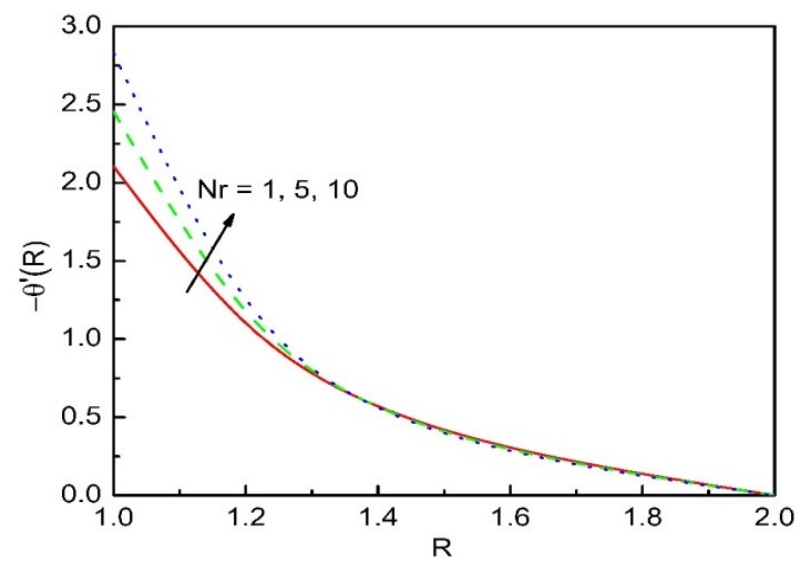

Figure 9: The influence of $\mathrm{Nr}$ on the temperature gradient

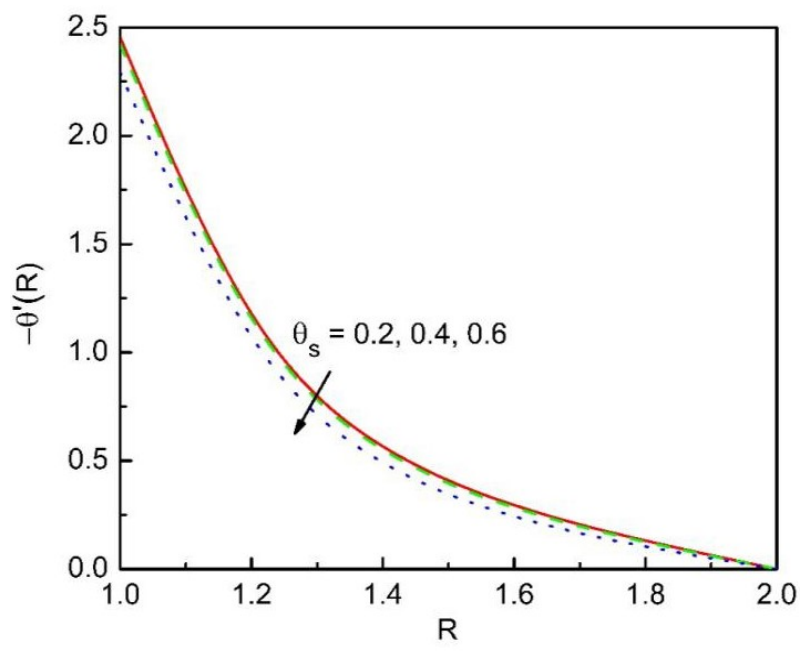

Figure 10: The influence of $\theta_{s}$ on the temperature gradient

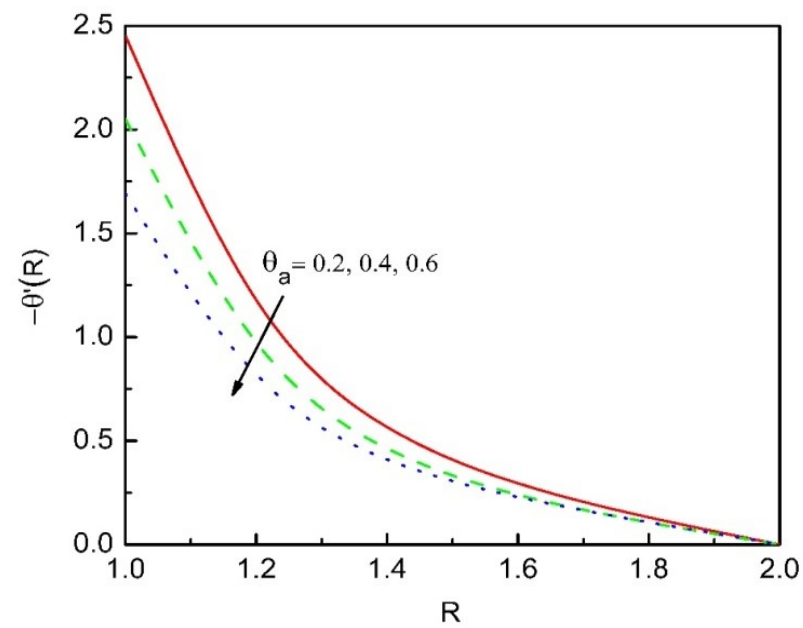

Figure 11 : The influence of $\theta_{a}$ on the temperature gradient 


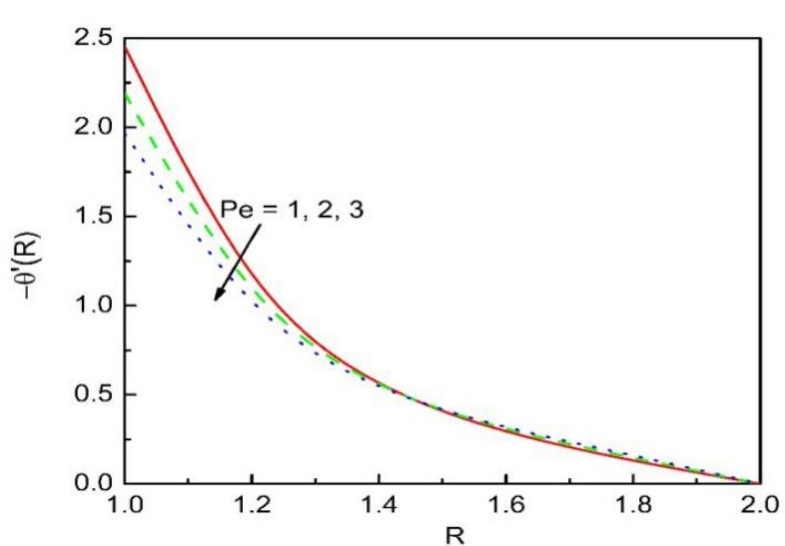

Figure $12:$ The influence of $P e$ on the temperature gradient

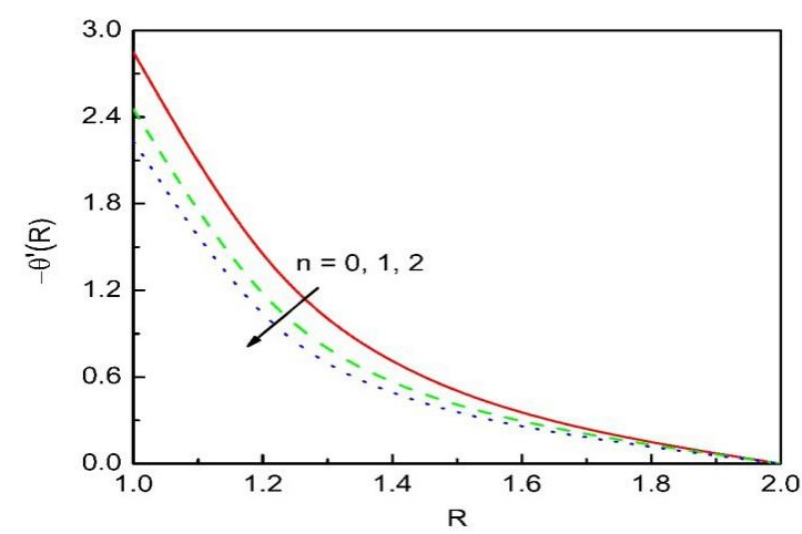

Figure $13:$ The influence of $n$ on the temperature gradient

\section{Conclusion}

The thermal analysis of the porous fin of the radial profile moving at constant speed is performed. Here, the heat transfer of the fin occurs by natural convection and the radiation conditions has been analyzed numerically with the help of Runge-Kutta Fehlberg fourth-fifth order method. The potted graphs were examined and it was found that the convective and radiative effect affects the temperature distribution of the fin. It is observed that the higher values of the convective parameter and the radiative parameter improve the rate of heat transfer from the fin surface. The thermal profile is lower for smaller values of the Peclet number and the exponent associated with the convective heat exchange coefficient. Increasing the temperature of the radiative and convective heat sink reduces the heat transfer rate of the fin.

\section{References}

1. Aziz, A., Khani, F., Analytic solutions for a rotating radial fin of rectangular and various convex parabolic profiles. Communications in Nonlinear Science and Numerical Simulation 15, 1565-1574, 2010.

2. Aziz, A., Khani, F., Convection-radiation from a continuously moving fin of variable thermal conductivity. Journal of the Franklin Institute 348, 640-651, 2011.

3. Darvishi, M.T., Gorla, R.S.R., Khani, F., Aziz, A., Thermal performance of a porous radial fin with natural convection and radiative heat losses. Thermal Science 19, DOI: 10.2298/TSCI120619149D, 2012.

4. Darvishi, M.T., Khani, F., Aziz, A., Numerical investigation for a hyperbolic annular fin with temperature dependent thermal conductivity. Propulsion and Power Research 5(1), 55-62, 2016.

5. Dogonchi, A.S., Ganji, D.D., Convection-radiation heat transfer study of moving fin with temperature-dependent thermal conductivity, heat transfer coefficient and heat generation. Applied Thermal Engineering 103, 705-712, 2016.

6. Hatami, M., Ahangar, GH.R.M., Ganji, D.D., Boubaker, K., Refrigeration efficiency analysis for fully wet semi-spherical porous fins. Energy Conversion and Management 84, 533-540, 2014.

7. Hatami, M., Ganji, D.D., Investigation of refrigeration efficiency for fully wet circular porous fins with variable sections by combined heat and mass transfer analysis. International Journal of Refrigeration 40, 140-151, 2014.

8. Khani, F., Darvishi, M.T., Gorla, R.S.R., Gireesha, B.J., Thermal analysis of a fully wet porous Radial fin with natural convection and radiation using the spectral collocation method. International Journal of Applied Mechanics and Engineering 21(2), 377-392, 2016.

9. Kim, T.Y., Kim, D.K., Kim, S.J., Scroll heat sink: A novel heat sink with the 
moving fins inserted between the cooling fins. International Journal of Heat and Mass Transfer 51, 3267-3274, 2008.

10. Ma, J., Sun, Y., Li, B., Simulation of combined conductive, convective and radiative heat transfer in moving irregular porous fins by spectral element method. International Journal of Thermal Sciences 118, 475-487, 2017.

11. Peng, H.S., Chent, C.L., Hybrid differential transformation and finite difference method to annular fin with temperature-dependent thermal conductivity. International Journal of Heat and Mass Transfer 54, 2427-2433, 2011.

12. Roy, P.K., Mallick, A., Mondal, H., Sibanda, P., A modified decomposition solution of triangular moving fin with multiple variable thermal properties. Arabian Journal for Science and Engineering 43, 1485-1497, 2018.

13. Torabi, M., Yaghoobi, H., Aziz, A., Analytical Solution for Convective Radiative Continuously Moving Fin with Temperature-Dependent Thermal Conductivity. International Journal of Thermophysics 33, 924-941, 2012.

14. Turkyilmazoglu, M., Heat transfer from moving exponential fins exposed to heat generation. International Journal of Heat and Mass Transfer 116, 346-351, 2018.

\section{GREEK SYMBOLS:}

$P=$ Density of the ambient fluid $\left(\mathrm{kg} / \mathrm{m}^{3}\right)$;

$\theta_{a}=$ Dimensionless convective sink temperature;

$\theta_{s}=$ Dimensionless radiative sink temperature;

$\theta=$ Non-dimensional temperature;

$\phi=$ Porosity;

$\sigma=$ Stefan-Boltzmann constant $\left(W / m^{2} K^{4}\right)$; and

$\varepsilon=$ surface emissivity of fin.

\section{NOMENCLATURE:}

$C_{\boldsymbol{p}}=$ Specific heat at constant pressure $(J / \mathrm{kgK})$;

$N c$ = Convective parameter;

$N r^{\circ}=$ Radiative parameter;

$P e=$ Peclet number;

$R \quad=$ Dimensionless radius;

$R^{*} \quad=$ Ratio of tip radius to base radius;

$T \quad=$ Local fin temperature $(K)$;

$T_{a}=$ Convective sink temperature $(K)$;

$T_{b}=$ Base temperature $(K)$;

$T_{g} \quad=$ Radiative sink temperature $(K)$;

$U=$ Constant velocity of the fin $(\mathrm{m} / \mathrm{s})$;

$h=$ Heat transfer coefficient $\left(W / m^{2} K\right)$;

$h_{b}=$ Heat transfer coefficient at temperature

$k_{\text {eff }}=$ Effective thermal conductivity of the material $(W / m K)$

$n=$ Exponent associated with the temperature dependent $h$;

$q=$ Base heat transfer rate $(W)$;

$r=$ Fin radius $(m)$;

$r_{b}=$ Base radius $(m)$;

$r_{t}=$ Tip radius $(m) ;$ and

$t \quad=$ Fin thickness $(m)$. 